\title{
LA RÉGULATION DES PROFESSIONS DE SANTÉ PAR LES ORDRES PROFESSIONNELS EN DROIT FRANÇAIS ET EUROPÉEN
}

The regulation of the health professions by professional councils in the Law of France and Europe

${ }^{1}$ Université de Nantes. Nantes/France.

E-mail: stephane.brissy@univ-nantes.fr.

Recebido em: 22/12/2015. Revisado em: 31/03/2016. Aprovado em: 04/04/2016. 


\section{RESUMÈ}

Cette contribution vise à analyser les formes que peut prendre le pouvoir normatif des ordres professionnels en santé en France et en droit de l'Union Européenne et les raisons pour lesquelles ce pouvoir est limité. Les ordres des professionnels de santé ont pour mission d'intégrer leur profession dans la société. Nous verrons que, par conséquent, l'activité des ordres professionnels est nécessairement subordonnée au contrôle de l'Etat mais que le champ de cette activité est aussi, de ce fait, plus large que celle d'un syndicat. Bien que limité par son objet même, le pouvoir normatif n'est néanmoins pas inexistant et nous verrons que ce pouvoir évolue et peut prendre des formes variées.

\section{Mots-clé}

Droit Européen; Droit Français; Ordres Professionels en Santé; Régulation des Professions de Santé.

\section{ABSTRACT}

This article aims to analyze the normative power of professional health councils in light of French and European law, highlighting the limits imposed on these organizations. Professional health councils have the mission of integrating their work into society, and their decisions are necessarily subject to State control, although the scope of the councils is broader than that of the unions. Despite being limited by its own purpose, the normative power of councils is present and evolving and taking on different forms.

\section{Keywords}

European Law; French Law; Health Professions Regulation; Professional Health Councils. 


\section{Introduction}

Le 9 avril 2015, lors d'une séance de débats à l'Assemblée Nationale française sur le projet de loi de modernisation du système de santé, 19 députés contre 10 décidèrent de voter la suppression de l'Ordre national des infirmiers crée neuf ans plus tôt. Même si lexistence de l'Ordre a été rétablie lors du passage du texte devant le Sénat, la difficulté pour de nombreux infirmiers à voir dans cet ordre un organe représentant la profession nécessite de s'interroger sur la pertinence d'une telle structure et sur l'importance de ses pouvoirs.

La reconnaissance législative d'un ordre professionnel a pourtant constitué une revendication importante des syndicats de professionnels, principalement de médecins, pendant le siècle qui a précédé la seconde Guerre mondiale ${ }^{1}$. Après avoir obtenu la légalisation de leur activité en 1892, les syndicats médicaux voyaient dans la création d'un ordre professionnel la véritable reconnaissance de l'identité et de l'autonomie de la profession médicale ${ }^{2}$. A leurs yeux, l'Ordre représentait en effet un moyen d'assurer une régulation de la profession en préservant ses principes moraux sans que l'Etat ne s'en charge lui-même. La création de l'Ordre devait ainsi s'accompagner de l'attribution à celui-ci d'un pouvoir normatif en lieu et place de celui de l'Etat.

La naissance de lordre des médecins a toutefois eu lieu dans un contexte troublé puisqu’elle a été décidée en 1940 par le gouvernement de Vichy, bien décidé à réguler lui-même les activités professionnelles pour y appliquer une politique ségrégationniste. L'ordre fut ainsi dans un premier temps en totale opposition à ses objectifs initiaux puisqu'il ne permettait ni d'assurer l'autonomie de la profession vis-à-vis de l'Etat ni de garantir sa dignité. La suppression de l'ordre puis son remplacement par une nouvelle instance ordinale en $1945^{3}$, aux côtés d'autres ordres de professionnels de santé, allait revenir sur cette soumission à l'Etat et tendre de nouveau à préserver léthique de la profession mais le pouvoir normatif des ordres allait être limité.

La gestion des politiques sociales a en effet été pensée comme un "champ d'action mixte" dans lequel interviennent les groupements professionnels et l'Etat pour garantir un équilibre Etat-société destiné à pacifier et démocratiser les relations sociales tout en permettant à l'Etat de jouer son rôle d'Etat-providence garant de droits sociaux fondamentaux ${ }^{4}$. En tant que composante des politiques sociales,

\footnotetext{
${ }^{1}$ OUILLARD, J. Historique du Conseil National de I'Ordre des Médecins (1845-1945). Histoire des Sciences Médicales, t. 39, n. 2, p. 213 et s., 2005. Disponible en: <http://www.biusante.parisdescartes.fr/sfhm/ hsm/HSMx2005x039x002/HSMx2005x039x002x0213.pdf>. Accédé en: 31 janv. 2017. L'établissement d'un projet de création d'un ordre des médecins en accord avec les syndicats médicaux et l'Académie de médecine date de 1929. Id. Ibid., p. 218.

${ }^{2}$ HASSENTEUFEL, P. Les médecins face à l'Etat: une comparaison européenne. Paris: Presses de Sciences Po., 1997.

${ }^{3}$ EVLETH, V. D. La bataille pour l'Ordre des médecins. Le Mouvement Social, n. 229, p. 61 et s., 2009.

${ }^{4}$ LE GOFF, J. Du silence à la parole: une histoire du droit du travail de 1830 à nos jours. Rennes: Press Univ. de Rennes, 2004. p. 283 et s. - DURKHEIM, E. De la division du travail social. Préface à la $2^{\mathrm{e}}$ éd. Paris: PUF, 2004. (Puf coll. Quadrige) - DIDRY, C. La réforme des groupements professionnels comme expression de la conception durkheimienne de l'Etat. Revue Française de Sociologie, v. 41, n. 3, p. 513 et s., jul./sep. 2000.
} 
la politique de santé suit elle aussi cet équilibre qui implique de donner à l'Etat un pouvoir normatif fort tout en laissant un champ d'intervention aux représentants des professionnels de santé. L’encadrement juridique des activités professionnelles a plusieurs sources et nombreuses sont aujourd'hui les règles de droit qui ne sont pas le fruit d'une action solitaire de l'Etat. Qu'ils soient partenaires de négociation, conseillers avisés que l'on consulte ou émetteurs de recommandations, les représentants des professionnels de santé interviennent de plusieurs manières dans le système normatif. Les ordres professionnels font partie de ces représentants mais les fonctions régulatrices que la loi leur attribue les apparentent plus à des organes de contrôle et de consultation quà des institutions permettant à une profession de créer ses propres règles.

Il serait cependant erroné d’assimiler les ordres professionnels à une institution étatique ou à l'inverse à une entité dont le but serait de défendre les intérêts d'une profession contre l'Etat. Il faut à mon sens dépasser cette vision binaire de la régulation des professions de santé et voir dans un ordre professionnel le représentant d'une profession dans sa globalité qui doit tendre à l'utilité sociale de la profession, autrement dit une cohésion entre le fonctionnement de la profession et celui de la société dans laquelle elle s'inscrit. L'ordre a une fonction sociale et un rôle de conciliation avec les pouvoirs publics ${ }^{5}$ qui limite son pouvoir régulateur. En cela les ordres ont une fonction spécifique qui les distingue des autres représentants professionnels, notamment syndicaux, ce qui n'exclut pas malgré tout une influence des ordres sur le contenu des normes.

\section{Une utilité sociale soumise au contrôle de l'Etat}

La création d’ordres professionnels de santé s'est très vite accompagnée d'une reconnaissance de leur rôle de coordination entre le fonctionnement de la profession et les intérêts de la société. L'observation faite au sujet de l'ordre des médecins qui, en contribuant à réguler la profession médicale assure une mission de service public $^{6}$, peut être étendue à tout ordre professionnel dans le domaine de la santé dont les missions ne sont guère différentes.

L'exercice d'une mission de service public relevant de la responsabilité de l'Etat, l'attribution d'une telle mission aux ordres professionnels ne pouvait se faire que sur délégation de l'Etat et sous son contrôle. Par leur participation à la régulation des professions de santé, les ordres apportent une connaissance des conditions d'exercice de l'activité professionnelle et sont censés faciliter l'acceptation de la régulation par les professionnels. La fonction des ordres n'est

\footnotetext{
${ }^{5}$ GLORION, B. L'Ordre des médecins: quels pouvoirs? Revue Pouvoirs, n. 89, p. 135-144, avr. 1999.

${ }^{6}$ CONSEIL D'ETAT. Assemblée, du 2 avril 1943, 72210. Disponible en: <https://www.legifrance.gouv.fr/ affichJuriAdmin.do?idTexte=CETATEXTO00007637163>. Accédé en: 31 janv. 2017.
} 
cependant pas de représenter les intérêts des professionnels mais de s'assurer que l'activité professionnelle ne sera pas exercée dans un sens contraire aux intérêts de la société, et notamment des patients. Le premier moyen pour y parvenir est de poser des règles déontologiques dont la fonction est de protéger les intérêts du patient, ceux des professionnels de santé et plus largement certaines valeurs éthiques propres à une profession et nécessaires à son utilité sociale, sa reconnaissance et son identité.

Le code de déontologie médicale français débute ainsi en affirmant les devoirs généraux des médecins qu'il distingue des devoirs envers les patients. Il affirme notamment que "le médecin, au service de l'individu et de la santé publique, exerce sa mission dans le respect de la vie humaine, de la personne et de sa dignité"?. La première mission de l'ordre des médecins est de veiller

au maintien des principes de moralité, de probité, de compétence et de dévouement indispensables à l'exercice de la médecine, de l'art dentaire, ou de la profession de sage-femme et à l'observation, par tous leurs membres, des devoirs professionnels, ainsi que des règles édictées par le code de déontologie ${ }^{8}$.

Des dispositions similaires concernent l'ordre des pharmaciens ${ }^{9}$, des infirmiers ${ }^{10}$ et des masseurs-kinésithérapeutes ${ }^{11}$, ajoutant à la préservation de l'indépendance et de la compétence des professionnels la promotion de la santé publique et de la qualité des soins.

Le rôle attribué par la loi aux ordres des professionnels de santé est ainsi expressément mis en relation avec les valeurs éthiques et sociales propres aux professions concernées et avec la politique de santé publique.

Ce lien explicite entre les missions des ordres et des objectifs dépassant les seuls intérêts des professionnels implique un contrôle de l'Etat, véritable garant du fonctionnement de la société et de ses valeurs. Le fondement même de la régulation professionnelle que constituent les règles déontologiques ne peuvent ainsi relever du seul pouvoir des ordres ${ }^{12}$. Ces derniers élaborent les règles déontologiques mais ne peuvent leur donner un caractère obligatoire qui nécessite l'intervention de l'Etat. Concernant par exemple la déontologie médicale, la loi prévoit ainsi "qu'un code de déontologie, propre à chacune des professions de médecin, chirurgien-dentiste

\footnotetext{
${ }^{7}$ Article R 4127-2 du Code de la santé publique (CSP). LEGIFRANCE. Code de la santé publique. Disponible en: <https://www.legifrance.gouv.fr/affichCode.do?cidTexte=LEGITEXT000006072665>. Accédé en: 31 janv. 2017.

${ }^{8}$ Article L 4121-2. Id. Ibid.

${ }^{9}$ Article 4231-1. LEGIFRANCE. Code de la santé publique, cit.

${ }^{10}$ Article L 4312-1 et 2. Id. Ibid.

${ }^{11}$ Article L 4321-14. Id. Ibid.

${ }^{12}$ GROSSET, M. Les logiques des normes sanitaires relatives à la pratique médicale. Journal de Droit de la Santé et de l'Assurance Maladie (JDSAM), n. 3, p. 51, 2013.
} 
et sage-femme, préparé par le conseil national de l'ordre intéressé, est édicté sous la forme d'un décret en Conseil d'Etat"13. Une règle déontologique ne peut être opposée à un professionnel ou par celui-ci si elle naa pas été validée par l'Etat sous la forme d'un décret, l'Etat restant par ailleurs libre de modifier le contenu du code de déontologie tel qu'il a été proposé par l'ordre.

La limitation du pouvoir de régulation par les ordres se retrouve également dans l'exercice de leur pouvoir disciplinaire. En effet, si les ordres professionnels exercent un contrôle disciplinaire sur l'activité de leurs confrères et peuvent les sanctionner professionnellement, ce pouvoir est limité par l'intervention de l'Etat. Non seulement l'organisation des juridictions disciplinaires et les peines qu'elles peuvent prononcer sont fixées par des règles étatiques. Mais en outre les décisions rendues par les instances ordinales peuvent faire l'objet d'un pourvoi en cassation devant le Conseil d'Etat, juridiction étatique qui peut remettre en cause l'interprétation de la loi faite préalablement par les juridictions disciplinaires. On peut ajouter que l'engagement d'une procédure disciplinaire devant la formation ordinale compétente n'empêche pas parallèlement la tenue d'une procédure devant les juridictions étatiques, l'action disciplinaire n'ayant qu'un champ limité dans ses fondements (une faute disciplinaire) comme dans ses conséquences (une sanction disciplinaire exclusive de toute réparation financière, notamment pour le patient).

Au niveau de l'Union Européenne, les règles régissant l'exercice des professions de santé sur lesquelles l'Union Européenne est compétente sont également fixées par des normes adoptées par les institutions de l'Union et non par des instances ordinales européennes. La reconnaissance des qualifications professionnelles obtenues dans un Etat membre par les professionnels de santé souhaitant travailler dans un autre Etat membre est ainsi opérée en application d'une directive européenne de $2005^{14}$. Les ordres des professionnels concernés ne peuvent créer de telles règles et si ceux-ci peuvent adopter des chartes déontologiques au niveau européen, comme l'a fait par exemple le Conseil européen de l'ordre des médecins ${ }^{15}$, il s'agit de règles non contraignantes pour les professionnels et les Etats membres.

La reconnaissance par l'Union Européenne de l'utilité d'une autorégulation n'empêche pas l'intervention de ses institutions. Le Parlement européen a certes reconnu que

\footnotetext{
${ }^{13}$ Article L 4127-1. LEGIFRANCE. Code de la santé publique, cit.

${ }^{14}$ EUR-LEX. Directive 2005/36/EC of the European Parliament and of the Council of 7 September 2005 on the recognition of professional qualifications. Disponible en: <http://eur-lex.europa.eu/legal-content/en/ TXT/?uri=CELEX\%3A32005L0036>. Accédé en: 31 janv. 2017.

${ }^{15}$ Charte européenne d'éthique médicale adoptée à Kos le 10 juin 2011 fixant 15 principes éthiques. CONSEIL EUROPÉEN ORDRES MÉDECINS. Charte Européenne d'Éthique Médicale, 10 juin 2011. Disponible en: <http://www.ceom-ecmo.eu/sites/default/files/documents/fr-charte_europeenne_dethique_medicaleadoptee_a_kos_0.pdf>. Accédé en: 31 janv. 2017.
} 
l'importance que revêtent l'éthique, la confidentialité à l'égard de la clientèle et un niveau élevé de connaissances spécialisées requiert l'organisation de systèmes d'autorégulation, tels ceux quétablissent actuellement les collèges et ordres professionnels ${ }^{16}$.

Mais ne nous y trompons pas, cette affirmation n'a nullement pour but de justifier un pouvoir décisionnaire des ordres professionnels affranchi d'un contrôle étatique. Elle s'accompagne immédiatement d'une volonté de libéraliser les services offerts par les professions libérales en supprimant les entraves à la libre concurrence sur le territoire européen. La référence à des valeurs professionnelles défendues par les ordres vise simplement à indiquer que l'ouverture à la concurrence de toutes les professions libérales doit se faire dans le respect des valeurs visées. La particularité de la position dégagée par le Parlement européen tient à la recherche d'une conciliation entre une autorégulation par les professions et la régulation par le simple jeu du marché. Elle montre que la seule régulation des activités professionnelles par les représentants d'une profession peut exposer les professions considérées aux ressorts économiques du marché, lesquels ne sont pas nécessairement compatibles avec les objectifs du droit de la santé et les finalités humanistes de l'activité des professionnels de santé. L'intervention de l'Etat pour garantir les droits sociaux que cette logique mercantile pourrait mettre en cause en est d'autant plus indispensable. Car c'est bien cette protection des droits fondamentaux qui justifie à la fois l'existence des ordres professionnels de santé et la limitation de leur pouvoir normatif.

En France, l'intervention de l'Etat a progressivement été étendue depuis le $19^{\mathrm{e}}$ siècle au-delà des seuls domaines régaliens que sont la sécurité et la justice. La confiance dans la capacité de la société à s'autoréguler, et des professions en particulier, a peu à peu laissé la place à un Etat-providence dont les fonctions sont de garantir certains droits, parmi lesquels des droits sociaux comme le droit à la santé et à une protection sociale.

La mise en place de règles déontologiques applicables aux professionnels de santé a certes conduit à mettre en place un ordre juridique professionnel mais un ordre juridique intégré à l'ordre étatique ${ }^{17}$. Et si l'Etat providence fait depuis quelques années l'objet de nombreuses attaques, le pouvoir normatif des ordres professionnels ne s'en trouve pas accru pour autant. Les conceptions parfois très différentes quant au rôle de l'Etat et à l'éthique entre les Etats membres de l'Union Européenne expliquent que les ordres professionnels de santé ne disposent pas non plus au sein de l'Union Européenne du pouvoir de créer des règles contraignantes.

\footnotetext{
${ }^{16}$ PARLEMENT EUROPÉEN. Proposition de résolution commune B5-0431, 11 décembre 2003. Disponible en: <http://www.europarl.europa.eu/sides/getDoc.do?pubRef=-//EP//NONSGML+MOTION+P5-RC-20030430+0+DOC+PDF+V0//FR>. Accédé en: 31 janv. 2017.

${ }^{17}$ MORET-BAILLY, J. Les déontologies. Marseille: Presses Universitaires d’Aix. 2001. p. 408 et s.
} 
Le rôle essentiel reconnu aux groupements professionnels en général dans lélaboration des règles de droit applicables aux activités professionnelles a pour but d'aider l'Etat à penser le social de manière pragmatique sans pour autant lui enlever son pouvoir de décision ${ }^{18}$.

L'intervention des pouvoirs publics français et européen dans la régulation des professions de santé n'exclut pas la reconnaissance d'un pouvoir normatif aux représentants de ces professions. Les ordres professionnels doivent à cet égard être distingués des organisations syndicales qui disposent certes d'un pouvoir normatif plus contraignant mais aussi de missions différentes.

\section{Une fonction sociale différente de l'activité syndicale}

Avant même que les ordres professionnels de santé n’apparaissent, les organisations syndicales revendiquaient le droit de créer des règles propres à leur profession. Ce pouvoir leur a été progressivement et en partie reconnu par la possibilité de négocier et de conclure des conventions ayant valeur de règles de droit applicables aux professionnels visés par la norme ainsi adoptée. Le statut des professionnels ayant la qualité de salariés travaillant sous les ordres et pour le compte d'un employeur privé est en partie déterminé par des conventions et accords collectifs de travail. Quant aux professionnels de santé libéraux, c'est-à-dire travaillant pour leur propre compte, leurs relations avec l'assurance maladie et les règles tarifaires notamment sont fixées par des conventions nationales ou départementales négociées entre les organisations syndicales représentatives et les institutions gérant le système de l'assurance maladie.

Ces règles s'appliquent à tous les professionnels dont l'activité entre dans le champ des normes conventionnelles, qu'ils soient ou non adhérents du ou des syndicats ayant conclu la convention. La seule réserve tient dans la faculté pour un professionnel libéral de refuser individuellement dêtre soumis à la convention nationale.

Comment expliquer ce déséquilibre dans le pouvoir normatif accordé à des représentants professionnels tous élus par leurs pairs que sont les syndicats et les ordres? La légitimité élective ne peut y suffire puisque les membres des ordres sont élus. Ce sont les différences dans les missions des uns et des autres qui vont nous permettre de comprendre cette apparente distorsion de compétence.

Les ordres professionnels ont avant tout pour mission d'assurer le respect de valeurs éthiques et sociales fondamentales propres à la profession qu'ils représentent, permettant par-là de faire reconnaître son utilité sociale et son identité. Les organisations syndicales ont en revanche un but plus restreint qui doit se limiter à "la défense des droits ainsi que des intérêts matériels et moraux, tant collectifs

\footnotetext{
${ }^{18}$ Pour un rapide résumé des théories développées en France sur la question, v. LE GOFF, J. op. cit., p. 284 et s.
} 
qu'individuels, des personnes mentionnées dans leurs statuts" ${ }^{\prime 1}$. Le pouvoir des syndicats de conclure des normes conventionnelles avec un employeur, des représentants d'employeurs ou les gestionnaires de l'assurance maladie est ainsi avant tout destiné à protéger les intérêts des professionnels contre ceux de lemployeur ou contre les pouvoirs publics dans une logique mêlant compromis et conflit social. La reconnaissance légale des conventions collectives dans le secteur privé visait à donner aux salariés une puissance collective de négociation face au pouvoir patronal. Quant au pouvoir conventionnel des syndicats de professionnels libéraux il a pour origine une volonté d'apaiser les professionnels en leur assurant qu'ils ne seront pas des salariés de l'assurance maladie soumis à ses directives mais des professionnels indépendants des pouvoirs publics et ayant le pouvoir de négocier collectivement leurs relations avec l'assurance maladie, notamment sur leurs tarifs ${ }^{20}$.

Les ordres ne sont pas un substitut aux syndicats mais un complément à ceuxci. Les organisations syndicales ne peuvent assumer la fonction d'inclusion sociale d'une profession de la même manière que les ordres, leur pouvoir normatif nétant pas étendu à lensemble de la profession représentée. Leur pouvoir de négociation est en effet limité à un mode d’exercice de l'activité. Les conventions conclues avec l'Assurance maladie ne concernent ainsi que les professionnels libéraux et non les professionnels salariés. Les conventions collectives et accords collectifs de travail ne visent quant à eux que les professionnels de santé salariés c'est-à-dire travaillant pour le compte d'un employeur. Le champ d'application des normes négociées par les syndicats de professionnels dépend ainsi de la façon dont le travail du professionnel est accompli, sous les ordres d'une hiérarchie pour les salariés ou de manière indépendante pour les professionnels libéraux. Le pouvoir normatif des syndicats ne sétend pas à lensemble de la profession alors que la fonction représentative des ordres concerne elle toute la profession, quel que soit son mode d'exercice. Les intérêts matériels et moraux des professionnels peuvent en effet être très différents suivant que le professionnel est salarié ou libéral mais les valeurs essentielles d'une profession ne peuvent en revanche quềtre communes. Tous les membres de la profession considérée, si elle est suffisamment importante pour être organisée au sein d’un ordre, doivent souscrire à ces valeurs jugées essentielles pour la profession et la société dans son ensemble, alors que les intérêts défendus par telle ou telle organisation syndicale peuvent ne pas être partagés par tous les professionnels. Cela explique que tous les membres d'une profession organisée en ordre doivent payer une cotisation à lordre alors que le principe constitutionnel de liberté syndicale empêche parallèlement d’obliger les professionnels à adhérer et à cotiser auprès d'un syndicat. Les différences d'intérêts entre membres d'une même

\footnotetext{
${ }^{19} \mathrm{Article} \mathrm{L}$ 2131-1 du Code du travail, texte applicable aussi bien aux syndicats de salariés qu'aux syndicats représentant les professions libérales. LEGIFRANCE. Code du travail, versión consolidée au 1 février 2017. Disponible en: <https://www.legifrance.gouv.fr/affichCode.do?cidTexte=LEGITEXT000006072050>. Accédé en: 31 janv. 2017.

${ }^{20}$ RÉGEREAU, M. La politique conventionnelle: ses ambitions et ses limites. Revue Française d'Administration Publique, n. 113, p. 76, 2005.
} 
profession expliquent également que les organisations syndicales appelées à négocier soient multiples et souvent d'avis divergents. Il n'existe en revanche qu'un seul ordre représentant une même profession de santé, quand bien même les différents discours syndicaux auraient réapparu au moment des élections ordinales.

Les différences entre lobjet des syndicats et celui des ordres professionnels de santé permettent de comprendre les attaques, voire l'animosité, dont les ordres sont parfois l'objet de la part des syndicats ou des professionnels eux-mêmes. Les ordres sont davantage assimilés à des organes de contrôle de la profession que sous-tend leur fonction de cohésion sociale. Il peut dès lors paraître paradoxal que les ordres ne disposent pas d'un pouvoir de créer les règles de droit applicables à leur profession alors que des syndicats aux missions plus restreintes peuvent participer plus directement à la création de certaines de ces règles. Certes, les syndicats qui négocient les conventions professionnelles doivent pour cela obtenir un score minimum au cours délections auxquelles votent la partie de la profession concernée par leur action ${ }^{21}$. Mais un ordre professionnel a lui aussi une légitimité électorale puisque l'ensemble de la profession concernée vote pour choisir les personnes qui vont le composer.

Cet apparent paradoxe peut être tempéré par une vision élargie de ce quest un pouvoir normatif. Dépassant une fonction simplement répressive vis-à-vis des professionnels, les prérogatives accordées aux ordres leur confèrent une influence qui peut être assimilée à une forme de pouvoir normatif.

\section{Une mission source d'influence normative}

Bien que ne pouvant créer eux-mêmes des règles de droit qui peuvent être sanctionnées directement devant un juge, qu'il soit ordinal ou étatique, les ordres professionnels de santé ne sont pas dépourvus d'un certain pouvoir normatif. S'ils ne créent pas de règle de droit "dur", c'est-à-dire explicitement assorties d'une sanction, ils n’en sont pas moins à l’origine d'un droit dit "souple" qui nest pas dépourvu de force normative ${ }^{22}$. Ce pouvoir, partagé avec de nombreuses autres institutions mais qui n'interviennent pas toujours dans le même domaine ${ }^{23}$, peut se manifester par l'expression d’opinions et d'avis que les pouvoirs publics sont parfois tenus de recueillir.

\footnotetext{
${ }^{21}$ Pour les salariés du secteur privé, un syndicat doit obtenir 10\% des suffrages exprimés aux élections professionnelles dans l'entreprise et 8\% dans sa branche professionnelle (articles L 2122-1 et L 2122-5 du Code du travail. LEGIFRANCE. Code du travail, versión consolidée au 1 février 2017, cit.). Les syndicats représentant les professionnels libéraux (non-salariés) ne peuvent négocier la convention nationale que s'ils ont obtenu au moins $10 \%$ des suffrages exprimés au niveau national aux dernières élections aux Unions régionales des professionnels de santé (article R 162-54-1 du Code de la sécurité sociale. LEGIFRANCE. Code de la sécurité sociale, versión consolidée au 12 février 2017. Disponible en: <https:// www.legifrance.gouv.fr/affichCode.do?cidTexte=LEGITEXTO00006073189>. Accédé en: 31 janv. 2017).

${ }^{22}$ THIBIERGE, C. et al. La force normative: naissance d'un concept. Paris: LGDJ, 2009.

${ }^{23}$ LE DOSSIER thématique du Journal de Droit de la Santé et de l'Assurance Maladie: les Normes en Santé: avis, recommandations, guides de bonne pratique. Journal de Droit de la Santé et de l'Assurance Maladie (JDSAM), n. 3, 2015.
} 
Signe de l'importance accordée au travail des ordres sur le contenu des règles déontologiques, les pouvoirs publics ne peuvent se contenter d'appliquer des règles professionnelles antérieures à la création de l'ordre lorsque ces règles entrent dans son domaine de compétence. Le Conseil d'Etat a ainsi condamné l'Etat français pour ne pas avoir encore adopté le code de déontologie préparé par l'ordre des infirmiers, quand bien même des règles issues d'un décret existaient déjà avant l'ordre luimême ${ }^{24}$. Une fois que la loi reconnaît l'existence de l'ordre et son pouvoir de préparer un corps de règles déontologiques, l'Etat ne peut plus se contenter des anciennes règles et doit en élaborer de nouvelles au regard du projet élaboré par l'ordre. Si l'Etat a bien le dernier mot, il doit ainsi prendre en considération les positions ordinales.

L'influence des ordres professionnels peut également se manifester par les avis qu'ils donnent suite aux nombreuses consultations obligatoires. Les pouvoirs publics doivent notamment consulter un ou plusieurs ordres avant d'autoriser un professionnel à exercer la médecine comme remplaçant ${ }^{25}$, à exercer en France lorsqu'il a obtenu son titre dans un autre Etat ${ }^{26}$ ou encore avant d'organiser les territoires de permanence des soins afférents à chaque département ${ }^{27}$.

La liste est encore longue mais le rôle consultatif des ordres professionnels n'est pas d’application générale et doit avoir été expressément prévu par la loi. A titre d'exemple, la détermination par un décret des conditions dans lesquelles le titre d'ostéopathe peut être utilisé ne nécessite pas la consultation du Conseil national de l'Ordre des médecins et du Conseil national de l'Ordre des masseurs-kinésithérapeutes si la loi ne l'impose pas expressément dans ce $\mathrm{cas}^{28}$. A l'inverse lorsque la loi impose de consulter un organisme représentant une ou des professions, qu'il s'agisse ou pas d'un ordre professionnel, l'absence de consultation rend le texte réglementaire illégal et dès lors susceptible d'annulation par le juge administratif ${ }^{29}$.

\footnotetext{
${ }^{24} \mathrm{CE}, 20$ mars 2015, $\mathrm{n}^{\circ} 374582$ (LEGIFRANCE. Disponible en: <https://www.legifrance.gouv.fr/affichJuriAdmin.do?id Texte=CETATEXTO00030445668 $>$. Accédé en: 31 janv. 2017). Il s'agit en l'occurrence des règles professionnelles issues du décret $n^{\circ}$ 93-221 du 16 février 1993, repris et modifié par un décret $n^{\circ}$ 2004-802 du 29 juillet 2004 (LEGIFRANCE. JORF $n^{\circ} 183$ du 8 Août 2004, p. 14150. Disponible en: <https://www.legifrance.gouv.fr/affichTexte. do?cidTexte=JORFTEXTO00000787339>. Accédé en : 31 janv. 2017). L'Ordre national des infirmiers a quant lui été créée en 2006 (LEGIFRANCE. JORF n²99 du 27 décembre 2006, page 19689. Loi n²006-1668 du 21 décembre 2006 portant création d'un ordre national des infirmiers.), et le projet de code de déontologie élaboré par l'ordre a été remis au ministre de la santé le 10 mars 2010. Le Conseil d'Etat a enjoint à l'État de prendre le décret nécessaire avant le 31 décembre 2015 sous astreinte de 500 euros par jour de retard.

${ }^{25}$ Article L 4131-2. LEGIFRANCE. Code de la santé publique, cit.

${ }^{26}$ Article L 4111-2. Id. Ibid.

${ }^{27}$ Article R 6315-6. Id. Ibid.

${ }^{28} \mathrm{CE}, 17$ juillet 2013, $\mathrm{n}^{\circ} 347291$ (LEGIFRANCE. Disponible en: <https://www.legifrance.gouv.fr/affichJuriAdmin. do?idTexte=CETATEXTO00027724291>. Accédé en: 31 janv. 2017). Sur la simple faculté de consulter l'ordre en cas de demande d'adhésion d'un professionnel à un protocole de coopération, v. CE, 20 mars 2013, $n^{\circ}$ 337577 (LEGIFRANCE. JORF $n^{\circ} 0071$ du 24 mars 2013, page 5015. Disponible en: <https://www.legifrance. gouv.fr/affichTexte.do?cidTexte=JORFTEXT000027206314>. Accédé en : 31 janv. 2017).

${ }^{29} \mathrm{Au}$ sujet d'un texte adopté sans consultation préalable du Haut conseil des professions paramédicales : CE, 17 juill. 2013, op. cit.; CE, 11 oct. 2010, n 329373 (LEGIFRANCE. Disponible en: <https://www.legifrance. gouv.fr/affichJuriAdmin.do?idTexte=CETATEXT000022931736>. Accédé en: 31 janv. 2017).
} 
Les avis et propositions formulés par l’ordre peuvent déterminer le contenu des normes adoptées par les pouvoirs publics, spécialement lorsqu'ils prennent la forme d'un énoncé normatif facilitant la tranposition de l'avis en règle de droit. Un code de déontologie préparé par l'ordre, par exemple, peut constituer bien plus qu'une source d'inspiration pour les pouvoirs publics qui peuvent être tentés de ne le modifier qu'á la marge dès l'instant oú aucune illégalité n’est constatée. Le "droit souple" peut dans ce cas fortement influencer le contenu du "droit dur".

La consultation des acteurs concernés par les normes adoptées est également très répandue au sein des institutions de l'Union Européenne. La Commission européenne a clairement exposé cette volonté de consulter les parties prenantes qui s'inscrit dans une démarche dite de "meilleure régulation"30. Les ordres regroupés au sein d’organisations telles que le Conseil européen de l'ordre des médecins ou le Comité permanent européen des médecins par exemple, peuvent ainsi formuler des avis et propositions sur des textes en préparation ou déjà en vigueur ${ }^{31}$.

Le rôle normatif de l'ordre est également très présent dans l'application des règles d'exercice de la profession. L’ordre est chargé de gérer les inscriptions au tableau de l'ordre, inscription sans laquelle un professionnel ne peut légalement exercer son activité. Enlever ce pouvoir à l'ordre, ou constater son incapacité à en assumer l'exercice constituent des signes forts de défiance à son égard, comme l'ont indiqué certaines organisations syndicales au sujet du nombre important d'infirmiers exerçant sans être inscrits au tableau de lordre national des infirmiers ${ }^{32}$.

Même une fois le professionnel inscrit, l'exercice de son activité peut être affecté par une décision ordinale de suspension temporaire, totale ou partielle, du droit d'exercer en cas d'insuffisance professionnelle ${ }^{33}$, ou d'infirmité ou d'état pathologique $^{34}$ lorsque l'une ou l'autre de ces situations rend dangereux l'exercice de l'activité.

Le pouvoir normatif peut également se manifester par une interprétation des normes étatiques, interprétation à laquelle les ordres peuvent procéder en proposant des modèles de contrats pour que les contrats conclus par les professionnels dans leur activité soient conformes à la loi.

\footnotetext{
${ }^{30}$ EUROPEAN COMMISSION. Better regulation for better results. Communication from the Commission to the European Parliament, the Council, the European Economic and Social Committee and the Committe of Regions. Strasbourg, 19.5.2015. $\operatorname{COM(2015)~} 215$ final. Disponible en: <http://ec.europa.eu/smartregulation/better_regulation/documents/com_2015_215_en.pdf>. Accédé en: 31 janv. 2017.

${ }^{31}$ V. P. Ex. La proposition d'amendements du CPEM à la proposition de directive modifiant la directive 2005/36 relative aux qualifications professionnelles, 27 mars 2012 (EUR-LEX. Directive 2005/36/EC of the European Parliament and of the Council of 7 September 2005 on the recognition of professional qualifications, cit.)

${ }^{32}$ Le Syndicat national des infirmières et infirmiers libéraux regrette la position trop tolérante du Ministère vis á vis des professionnels non-inscrits et la Fédération Nationale des Infirmiers déplore quant à elle la mise em péril des élections aux Unions Régionales des Professionnels de Santé qu'implique le décalage entre le nombre d'infirmiers déclarés auprès de l'assurance maladie et ceux, bien moins nombreux, inscrits au tableau de l'ordre.

${ }^{33}$ Article R 4124-3-5. LEGIFRANCE. Code de la santé publique, cit.

${ }^{34}$ Article R 4124-3. Id. Ibid.
} 
Le pouvoir d'interprétation est aussi le fait des juridictions disciplinaires lorsqu'elles doivent se prononcer sur l'existence d'une faute. Les formations disciplinaires des ordres sont libres de se déterminer sur les situations correspondant ou non à une violation de principes déontologiques comme l'indépendance professionnelle ou le secret professionnel par exemple. Un tel pouvoir est incontestablement une source normative, la norme ne provenant pas seulement de la règle énoncée mais aussi de l'application que son interprétation par un juge permet d'en faire.

Les normes différentes des normes étatiques et ordinales, telles que les recommandations de bonne pratique par exemple, sont toutefois de plus en plus nombreuses et marquent, sous couvert de leur pertinence scientifique, une véritable concurrence entre le pouvoir des experts scientifiques et celui de la communauté professionnelle ${ }^{35}$.

Il semble alors nécessaire pour les représentants d'une profession, et notamment pour les ordres, non pas de réclamer de nouveaux pouvoirs mais daccroître leur capacitéà relayer les opinions et les expériences des professionnels auprès des pouvoirs publics. Pour les raisons énoncées plus haut tenant à la fonction sociale des ordres, leur pouvoir normatif doit davantage se manifester par une participation à la réflexion en amont et en aval de lélaboration des règles encadrant les activités professionnelles. Linfluence d'un ordre sur le contenu de ces règles ne sera que plus grande s'il peut faire valoir auprès des pouvoirs publics un recueil élargi de lopinion des professionnels représentés. La grande consultation lancée en octobre 2015 par le Conseil national de lordre des médecins sur lavenir du système de santé en est un exemple. En vue d’enrichir la réflexion sur le système de santé, le CNOM a mis en place un site internet ${ }^{36}$ récapitulant les rencontres publiques organisées à cet effet et contenant un questionnaire dans lequel les médecins peuvent exprimer leurs opinions sur les évolutions du système et le rôle que pourraient y jouer le médecin. L’objectif clairement affiché est de transmettre les résultats de cette grande consultation aux pouvoirs publics pour que les opinions du corps médical soient prises en compte, la consultation devant déboucher sur un rapport servant de socle à des propositions dévolution du système de santé.

Cet exemple récent rappelle que la limitation du pouvoir normatif des ordres professionnels ne signifie pas que ce pouvoir est inexistant. Il peut être dautant plus important si les avis et propositions formulés reposent sur une consultation des professionnels euxmêmes et non uniquement sur le mandat octroyé à lordre par lélection. Cest sans doute en élargissant le recours à des procédés de démocratie participative, notamment via internet, en leur sein et en encourageant leur géneralisation que les ordres professionnels pourront faire jouer son plein effet à leur pouvoir normatif. Cést peut-être aussi le concept de démocratie participative qui correspond le mieux à lessence même d’un ordre professionnel qui est de mettre en lien une communauté professionnelle avec la société dans son ensemble.

\footnotetext{
${ }^{35}$ TABUTEAU, D. Référentiels, bonnes pratiques et recommandations: nouvelles normes ou "quasi normes" en santé? Journal de Droit de la Santé et de l'Assurance Maladie (JDSAM), n. 3, p. 16, 2015.

${ }^{36}$ CONSEIL NATIONAL DE L'ORDRE DES MÉDECINS. Disponible en: <http://www.lagrandeconsultation.medecin.fr/>. Accédé en: 31 janv. 2017.
} 


\section{References}

CONSEIL D’ETAT. Assemblée, du 2 avril 1943, 72210. Disponible en: <https://www.legifrance. gouv.fr/affichJuriAdmin.do?idTexte=CETATEXT000007637163>. Accédé en: 31 janv. 2017.

CONSEIL NATIONAL DE L'ORDRE DES MÉDECINS. Disponible en: <http://www. lagrandeconsultation.medecin.fr/>. Accédé en: 31 janv. 2017.

DIDRY, C. La réforme des groupements professionnels comme expression de la conception durkheimienne de l'Etat. Revue Française de Sociologie, v. 41, n. 3, p. 513-538, jul./sep. 2000.

DURKHEIM, E. De la division du travail social. Préface à la 2e éd. Paris: PUF, 2004. (Puf coll. Quadrige).

EUROPEAN COMMISSION. Better regulation for better results. Communication from the Commission to the European Parliament, the Council, the European Economic and Social Committee and the Committe of Regions. Strasbourg, 19.5.2015. COM(2015) 215 final. Disponible en: $<$ http://ec.europa.eu/smart-regulation/better_regulation/documents/com_2015_215_en.pdf > . Accédé en: 31 janv. 2017.

EVLETH, V. D. La bataille pour l'Ordre des médecins. Le Mouvement Social, n. 229, p. 61-77, 2009.

GLORION, B. L'Ordre des médecins: quels pouvoirs? Revue Pouvoirs, n. 89, p. 135-144, avr. 1999.

GROSSET, M. Les logiques des normes sanitaires relatives à la pratique médicale. Journal de Droit de la Santé et de l'Assurance Maladie (JDSAM), n. 3, 2013.

HASSENTEUFEL, P. Les médecins face à l'Etat: une comparaison européenne. Paris: Presses de Sciences Po., 1997.

LE DOSSIER thématique du Journal de Droit de la Santé et de l'Assurance Maladie: les Normes en Santé: avis, recommandations, guides de bonne pratique. Journal de Droit de la Santé et de l'Assurance Maladie (JDSAM), n. 3, 2015.

LE GOFF, J. Du silence à la parole: une histoire du droit du travail de 1830 à nos jours. Rennes: Press Univ. de Rennes, 2004.

MORET-BAILLY, J. Les déontologies. Marseille: Presses Universitaires d’Aix. 2001.

POUILLARD, J. Historique du Conseil National de l'Ordre des Médecins (1845-1945). Histoire des Sciences Médicales, t. 39, n. 2, 2005. Disponible en: <http://www.biusante.parisdescartes.fr/ sfhm/hsm/HSMx2005x039x002/HSMx2005x039x002x0213.pdf>. Accédé en: 31 janv. 2017.

RÉGEREAU, M. La politique conventionnelle: ses ambitions et ses limites. Revue Française d'Administration Publique, n. 113, p. 75-82, 2005.

TABUTEAU, D. Référentiels, bonnes pratiques et recommandations: nouvelles normes ou "quasi normes" en santé? Journal de Droit de la Santé et de l'Assurance Maladie (JDSAM), n. 3, 2015. 
La régulation des professions de santé en France et Europe

THIBIERGE, C. et al. La force normative: naissance d'un concept. Paris: LGDJ, 2009.

\section{Remerciements}

Cette étude a été rendue possible dans le cadre de la coopération académique entre le Centre de Recherche en Droit de la Santé de l'Université de São Paulo (NAP-DISA/USP) et le Institut Droit et Santé de l'Université de Paris 5 et grâce au financement fourni par le Programme USP-COFECUB.

Stéphane Brissy - Maître de conférences à l'Université de Nantes. Membre de l'Institut Droit et Santé de l'Université Paris Descartes; INSERM UMR S 1145. Nantes, France. E-mail: stephane.brissy@univ-nantes.fr. 